\title{
Una mirada a la creatividad del docente desde una práctica pedagógica reflexiva
}

\author{
A look at the creativity of the teacher \\ from a reflective pedagogical practice
}

DOI: http://dx.doi.org/10.17981/cultedusoc.10.2.2019.11

Recibido: 27/08/2019 Aceptado: 26/11/2019

Aliety Sosa Padrón

Universidad Autónoma de Chihuahua (México)

p33611@uach.mx

Para citar este artículo:

Sosa, A. (2019). Una mirada a la creatividad del docente desde una práctica pedagógica reflexiva. Cultura, Educación y Sociedad, 10(2). 137-146. DOI: http://dx.doi.org/10.17981/cultedusoc.10.2.2019.11

\section{Resumen}

El artículo tiene como objetivo analizar la creatividad del docente en el contexto de enfoques teóricos emergentes como la práctica pedagógica reflexiva. Metodológicamente se abordan dos momentos de investigación: en primer lugar se trabaja con la técnica de la revisión y análisis documental para develar el tratamiento que los autores hacen del concepto de creatividad en función de la práctica reflexiva del docente. En segundo término se desarrollan sesiones de trabajo, tipo seminario - taller donde se conforman grupos de discusión, con docentes del área de Filosofía y Letras de la Universidad de Chihuahua, México, quienes en construcción colectiva se aproximan a un concepto colegiado de creatividad; el concepto es validado a través de la observación de los grupos de discusión en atención al acompañamiento en procesos de: planeación didáctica- intervención pedagógica- evaluación instruccional. Los resultados destacan competencias docentes, asociadas a su creatividad para la mediación didáctica desde una postura reflexiva, donde se identifican fortalezas y vacíos por resolver. Se concluye en la necesidad de consolidar una educación creativa sustentada en la resignificación de la práctica pedagógica, en función de los modos de actuación del docente y en el marco de las transformaciones sociocontextuales.

Palabras clave: Creatividad; práctica pedagógica reflexiva; formación docente

\section{Abstract}

The article aims to analyze the creativity of the teacher in the context of emerging theoretical approaches such as reflective pedagogical practice. Methodologically, two moments of research are approached: firstly, we work with the technique of the review and documentary analysis to reveal the treatment that the authors make of the concept of creativity based on the reflective practice of the teacher. Secondly, work sessions are developed, such as seminars - workshops where discussion groups are formed, with teachers from the area of Philosophy and Letters of the University of Chihuahua, Mexico, who in collective construction approach a collegial concept of creativity; The concept is validated through the observation of the discussion groups in support of the accompaniment in processes of: didactic planning - pedagogical intervention - instructional evaluation. The results highlight teaching competencies, associated with their creativity for didactic mediation from a reflexive position, where strengths and gaps to be resolved are identified. It concludes the need to consolidate a creative education based on the resignification of pedagogical practice, depending on the teacher's ways of acting and within the framework of socio-contextual transformations.

Keywords: Creativity; reflective pedagogical practice; teacher training 


\section{INTRODUCCIÓN}

El presente artículo forma parte de una investigación más amplia, dentro de la maestría en Innovación Educativa en la Facultad de Filosofía y Letras de la Universidad Autónoma de Chihuahua. Su objetivo ha sido acercarse a la creatividad del docente desde la perspectiva de una práctica pedagógica reflexiva. Se intenta develar el tratamiento teórico que diferentes autores desarrollan del constructo creatividad; este abordaje teórico se hace en función de la caracterización de la práctica docente y su necesario acompañamiento hacía modos de actuación donde la creatividad y la inventiva sean rasgos esenciales.

La educación entendida desde la creatividad, supone reconocer las potencialidades que tienen los actores, especialmente el docente, de construir espacios para la creación de oportunidades en función de la resolución de problemas que surjan en sus espacios diarios de coexistencia. La realidad y su dinamismo, conlleva la necesidad de la interacción permanente con otros sujetos y con los objetos culturales propios de los contextos donde el docente interviene. De tal forma que en la sociedad actual, y más concretamente los retos a los que se enfrenta el sistema educativo, están referidos a cómo consolidar perfiles profesionales docentes donde se evidencie la creatividad, así como también otras cualidades como la innovación y el emprendimiento.

Fomentar y desplegar la creatividad, implica la movilización de la cultura, tradiciones, la valoración cualitativa del talento humano (Caballero, 2012); todas estas iniciativas conllevan al logro de una formación integral centrada en el despliegue de valores, actitudes, habilidades y competencias de desempeño en correspondencia con lo sociocontextual. Se infiere la necesidad de que la formación evidencie la creatividad como una condición inherente al currículo; todo ello validado por la necesidad de innovar y emprender como factores clave de desarrollo tanto humano, como social (Mujica, Marín, Smith y Lovera, 2008)

Entre los sujetos del proceso pedagógico que tienen un rol relevante en el desarrollo de la creatividad y de la innovación educativa, se distingue el docente por su responsabilidad para que los estudiantes tengan un papel protagónico en todas las actividades desarrolladas durante su formación. La idea expuesta traduce la necesidad de la creatividad docente permita aplicar estrategias didácticas para fortalecer en los estudiantes competencias, por ejemplo asociadas al manejo de la tecnología, de forma tal que su uso consciente y responsable, contribuya con el mejoramiento de su calidad de vida y desempeño académico. Así mismo, la creatividad en el acto docente permitirá al estudiante ser consciente de la importancia delos avances científicos en el marco de procesos como la globalización e internacionalización.

Varias son las investigaciones que han trabajado la creatividad y su papel en el proceso de enseñanza-aprendizaje; destacan los aportes de Martínez (2009), al considerar la creatividad como una condición susceptible de ser desarrollada, a través de los procesos pedagógicos. Implica fortalecimiento de los sujetos en sus componentes individuales y grupales como respuesta a la necesidad de innovar en el proceso educativo. En este orden coindicen, Csikszentmihalyi (1998), Chibás-Ortiz (2012) y Remedios (2016) cuando señalan que la creatividad del docente, además de ser una expresión de la personalidad desde su función de autoregulación, puede asociarse a factores extrapersonales como: la situación social contextual en que está inmerso el 
sujeto, la historicidad y el estado del conocimiento científico.

Del mismo modo se reconocen las posibilidades reales que tiene el sujeto para incrementar sus motivaciones; el planteamiento y solución de problemas es una importante vía para estimular la creatividad del docente. En cuanto al comportamiento creativo del docente está fuertemente imbricado por su desarrollo cognitivo y por la afectividad; en este sentido, los precitados autores coinciden es aceptar que los sujetos alcanzaran mayores niveles de desarrollo en aquellas áreas donde se concentran tendencias motivacionales como la originalidad, fluidez y flexibilidad en sus formas de actuación, lo que pasa a representar indicadores del comportamiento creativo de los docentes en su desempeño profesional.

Los argumentos expuestos forman parte del marco referencial de sustento al presente artículo; se analiza el desarrollo del pensamiento creativo a la luz de una práctica pedagógica reflexiva e innovadora. Existe la percepción generalizada de que en el contexto de la educación superior, en diferentes programas académicos, incluidos los orientados a la formación de docentes, el ciclo didáctico privilegia la actuación por rutinas, concibiendo un docente que lejos de asumir la innovación y creatividad, centra su desempeño en la trasmisión de información y contenidos asociados a los programas de las asignaturas, y donde emerge la necesidad de aprovechar las potencialidades de los estudiantes para la mediación didáctica en función de la resolución de problemas contextuales. Igualmente, se infieren vacíos en los procesos de planeación académica, así como la necesidad de incorporar actividades que contribuyan a desarrollar competencias científicas.

Los descriptores también perciben el uso de métodos y tareas docentes reproduc- tivas, la existencia de dificultades en los aprendizajes de los estudiantes que tienen su base en el predominio de la reproducción y el limitado aprovechamiento del pensamiento creativo, el empleo de estilos de comunicación autocráticos, predominio de un clima poco participativo en las actividades académicas, laborales, investigativas, donde no se asumen riesgos para romper las reglas establecidas, los resultados de los proyectos de investigación se usan solo en ocasiones para mejorar las prácticas pedagógicas.

\section{FUNDAMENTACIÓN TEÓRICA}

Se inicia la revisión teórica, analizando importantes investigaciones sobre la creatividad. Se considera oportuno mencionar la mirada de la psicología cognitiva que al investigar la creatividad hace importantes aportes a la comprensión del tema. En la generalidad de los casos se asumen que los cognitivistas centran la atención en un ser humano activo con su experiencia para procesar información en relación al contexto y al ambiente. A lo largo de varias investigaciones. En este maco referencial, De Bono (1986), propone como elementos esenciales del pensamiento creativo los siguientes: libertad de expresión, ausencia de inhibiciones, evitación de juicios críticos valorativos y estimulación de nuevas ideas durante el proceso creativo. Desde la perspectiva del autor el estudio del pensamiento creativo va asociado a técnicas que favorecen la capacidad de análisis y síntesis, así como también a la aplicación del razonamiento lógico, como base para la toma de decisiones.

En la misma línea de De Bono (1986), Guilford y Strom (1978) y Csikszentmihalyi (1998) dan preponderancia en el análisis de la creatividad, a los factores 
cognitivos (percepción y la elaboración de la información), afectivos (apertura a la experiencia, tolerancia a la ambigüedad, autoestima positiva, voluntad de obra y motivación de crear) y ambientales (las condiciones del contexto tanto físico como social que facilitan el desarrollo y la actualización del potencial creativo) en el acto creativo.

Por otra parte, Betancourt (1997) en el análisis que realiza acerca de los enfoques para definir el concepto creatividad (proceso, persona, producto, medio) profundiza en las características de la personalidad creadora a partir de los aportes de diferentes autores, lo que le permite concluir que la originalidad, la toma de decisiones flexibles, el uso de conocimientos existentes como base para nuevas ideas, la sensibilidad ante los problemas, la riqueza en sentimientos y emociones y la independencia, son los principales rasgos asociados al desarrollo de la creatividad en los sujetos. Estos aportes resultan fundamentales cuando se intenta explicar la creatividad asociada a la práctica reflexiva del docente.

Al respecto, Martínez (2009) argumenta que la actividad pedagógica es creadora por esencia, porque encierra, a través de sus componentes, cómo en el proceso docente educativo es posible formar la personalidad del estudiante de acuerdo con los objetivos sociales y en correspondencia con la actuación del maestro. Por su parte, Remedios (2016) asevera que para propiciar el desarrollo de la creatividad en el docente, debe prestarse especial atención al carácter creador de la actividad pedagógica y a las particularidades que adquiere el modo de actuación profesional desde las exigencias del objeto de la profesión, en correspondencia con el aprendizaje del estudiante. Esto se corresponde con lo expuesto por Cárdenas y Ángulo (2016), cuando destacan la importancia de la adaptación, mejoramiento e innovación como parte de los procesos de aprendizaje.

En este sentido, Chibás-Ortiz (2012) concibe una gestión educacional creativa significa a través de la implementación de estrategias flexibles que se adapten a la situación de la organización del proceso pedagógico y a su contexto, así como aplicar la gestión de proyectos donde el docente tiene un importante papel.

Por ende en los estudios en creatividad es necesario acercarse a los elementos que convergen y que invitan a ver la creatividad inserta en el sistema, con una mayor influencia sociocultural, ambiental o ecológica. Autores como Amabile (1983), Sternberg y Lubart (1997) y Csikszentmihalyi (1998), muestran que la creatividad va dejando de ser una capacidad personal para convertirse en un bien social.

La creatividad ha sido ubicada dentro de muchas realidades de la humanidad, erróneamente vinculada solo a las artes, separada de otras ciencias de las cuales no solo forma parte, sino que la necesitan. Torrance () definió la creatividad como un proceso; un proceso que permite estar atento a los problemas, falta de elementos o puntos «ciegos» en el conocimiento; encuentra esas dificultades y por lo tanto desarrolla soluciones, suposiciones o hipótesis; prueba y vuelve a probarlos; cambiar y volver a probarlos y comunicar los resultados.

En los tiempos actuales la educación quizás no ha sido más creativa porque se ha acomodado al medio, a diferentes procesos de adaptación, de socialización y hasta por la organización institucional. Castillejo, et al. (2012) señala que las prácticas escolares, incluso las buenas prácticas, están condicionadas por presiones de conformidad, de ajuste cerrado a patrones "de buena conducta" o de "rendimientos esperados". 
Sin embargo, una educación de calidad requiere que se produzca en los espacios educativos, no una simple reproducción, sino una renovación constante de los hábitos y prácticas sociales.

Por ello, Monsalve (2015) determina que las actividades en las aulas tienen que moverse más sobre los procesos de innovar que de renovar y no pueden menos que inspirarse en la creatividad; en la del educador y en los estudiantes mismos. Consecuentemente, la creatividad puede verse como un proceso complejo por las implicaciones conceptuales, actitudinales y procedimentales que conlleva; de allí que puede suponer relaciones inter y transdisciplinarias como vía para la comprensión del fenómeno educativo. Maldonado (2014), asocia el carácter complejo de la realidad en sí misma como determinante de los sistemas y subsistemas sociales.

Desde los aportes de Archila (2013) resulta importante considerar un nuevo paradigma que centre la discusión en la creación educativa, una educación que estimula la incertidumbre, lo diverso y el sentido de la recursividad e iteratividad. La creatividad así entendida privilegia el sentido de integración y encuentro entre disciplinas, propone un modo de pensar transdisciplinario, defendiendo su condición de unicidad en inevitable relación con el sujeto que piensa y con multiplicidad de formas culturales convergentes.

Asumir esta tarea en el plano educativo desde la transdisciplinariedad, implica un primer momento de ensayo, y/o adaptación en la interdisciplinariedad (López, 2015). Puesto que la transdisciplinariedad debe partir de una cimentación donde participen, jugando su rol, maestros, familia y estudiantes. Debe convertirse en la lucha contra el reduccionismo y la intolerancia a un aprendizaje diferente.
Para Schön (2010) la creatividad concebida como práctica reflexiva del docente implica aproximarse a la realidad desde la investigación; a decir del autor, no se trata solo de transferir lo aprendido, es necesario desarrollar la criticidad, indagar, analizar y experimentar, con la intención de reconceptualizar el proceso didáctico en atención al cuestionamiento de los esquemas de funcionamiento, así como también de las teorías implícitas en el proceso educativo. El autor caracteriza a la práctica docente y reflexiva como constructo complejo y de gran incertidumbre; otros principios intrínsecos al constructo están referidos a la inestabilidad, la singularidad y el conflicto de valores. En este orden de ideas Morin (2003) concibe la práctica docente como acción dentro del aula, pero desde una concepción amplia que atiende a las múltiples relaciones y sinergias que se dan dentro del ámbito educativo.

En consideración de Dewey (1933) la práctica reflexiva no significa que los maestros hayan de reflexionar sobre todo y durante todo el tiempo; es evidente que una postura totalmente contemplativa no es adecuada ni posible para los docentes. Esta posición, más bien, busca un equilibrio entre la reflexión y la rutina; entre el pensamiento y la acción.

\section{Metodología}

El diseño metodológico se fundamenta en la revisión y análisis documental. Se aborda un componente teórico para abordar las categorías centrales del estudio: Creatividad del docente y práctica pedagógica reflexiva, lo que cimienta las bases para desarrollar un proceso de argumentación. A través de la vía deductiva, se trasciende la realidad y se establecen relaciones conceptuales entre los constructos identificados. Con base a los aportes de Cisterna (2005) y Marín, Cabas, Cabas y Paredes (2018), se establecen como 
criterios para seleccionar las unidades teóricas de análisis, la pertinencia de la fuente consultada en cuanto provee información de impacto para explicar cada constructo, así como también su relevancia para develar la importancia de la temática trabajada.

El análisis documental se complementó con la técnica de grupos de discusión, a través de actividades tipo seminario - taller con docentes de la carrera de Historia, de la Facultad de Filosofía y Letras, de una Universidad pública. En estas sesiones se sugiere seguir una lógica de construcción conceptual, donde se invita al docente a repensar su práctica de una manera comprensiva, con el objetivo, no de cuestionarse duramente, sino de aprender de su misma experiencia, con la intención de aprender a aprender. De esta manera, se considera construirlo a partir de los siguientes interrogantes:

1. ¿Qué es?

2. ¿Para qué es, con qué fines?

3. ¿Qué implica, qué requiere para lograr este fin?

Los precitados cuestionamientos sirven de base para que los participantes, desde la discusión, argumentación y construcción en forma colegiada, se aproximen a una definición de creatividad del docente en correspondencia con su práctica pedagógica reflexiva; la elaboración del constructo se presenta en el apartado de los resultados. El acercamiento a la teoría permite tener claridad sobre los elementos que contribuyen a su enriquecimiento, con el propósito de que se evidencie el sentido innovador dentro del salón de clases.

La formación del docente de dicha carrera, sus contextos y experiencias, no solo en el aula, sino en su vida diaria, su compromiso con un alumno, que por el perfil que estudia, demanda un alto nivel de prepara- ción, conllevan a la necesidad de investigar acerca de su práctica pedagógica. Por ello, es importante considerar cuatro vertientes o elementos que sintetizan lo discutido acá: diálogo, trabajo colegiado, interdisciplina y transdisciplina, como componentes asociados al perfil docente, con el propósito de estar a tono con lo que la creatividad reflexiva demanda.

Las sesiones de trabajo se realizan en un clima, donde prevalece la necesidad de acompañamiento al docente, donde los cuestionamientos no son los que guían los encuentros; el docente representa el actor clave y no un objeto observado. El propósito no es juzgarlo como sujetos de una investigación, sino incluirlos en una automirada de su proceso docente, de manera tal que se mejore su práctica pedagógica

Estas sesiones conllevan un segundo momento. La construcción del concepto de manera colegiada permite la elaboración de una pauta de observación orientada a maestros innovadores, con la intención de ser congruente no solo con un estilo de trabajo, sino con los maestros que participan de estas sesiones y que permiten ser observados; además se operacionaliza el concepto construido, en función de los elementos implicados: diálogo, trabajo colegiado, interdisciplina y transdisciplina, determinando qué se pretende hacer, cómo se realiza y para qué se realiza.

Dicha pauta se divide en tres momentos: la planeación de la clase, la intervención en el aula y la evaluación de la clase, convirtiéndose en el procedimiento para la caracterización de la práctica docente. Dicha observación se realiza mediante videograbación. Para ello además se toman en cuenta los criterios manejados a partir de la tipificación realizada, la información de los coordinadores y los maestros que forman parte de las áreas estudiadas. 


\section{Resultados Y DISCUSIÓN}

De esta manera, se considera construirlo a partir de las siguientes interrogantes:

1. ¿Qué es?

2. ¿Para qué es, con qué fines?

3. ¿Qué implica, qué requiere para lograr este fin?

Los precitados cuestionamientos sirven de base para que los participantes, desde la discusión, argumentación y construcción en forma colegiada, se aproximen a una definición de creatividad del docente en correspondencia con su práctica pedagógica reflexiva

En atención a los interrogantes centrales en los grupos de discusión acerca de lo qué es, para qué es, con qué fines, qué implica, qué requiere, se generan acuerdos en función de conceptualizar la creatividad del docente en correspondencia con su práctica pedagógica reflexiva, dicha definición se expone a continuación:

"Proceso de descubrimiento o producción de algo nuevo, donde se desarrolla una enseñanza comprensiva, mediada por la reflexión de un conocimiento multidimensional, que borra un conocimiento específico, asociando la formulación, surgimiento, búsqueda y la solución de problemas, formando personas ricas en originalidad, flexibilidad, visión, iniciativa y confianza; personas amantes de los riesgos y listas para afrontar los obstáculos y problemas que se presenten en sus vidas; que ofrece herramientas para la innovación donde participan maestros $\mathrm{y}$ estudiantes como sujetos protagonistas, teniéndose en cuenta diferentes contextos y realidades inacabadas, la unidad sin borrarse la diversidad, asumiendo la interdependencia, el intercambio, la incertidumbre y las experiencias desde la visión sistémica del mismo"
En este sentido, el acercamiento a la práctica docente, a partir de la pauta de observación, sintetizada en el concepto previo, puso en evidencias diferentes resultados, que se describen a continuación:

Entre los resultados destaca que la planeación de la clase busca la interacción alumno-maestro, al tener en cuenta por parte del maestro, los miedos de sus alumnos, empleando estrategias para dar inicio a la misma y desarrollarla, a través del debate, buscando ser original al generar ideas propias y ser flexible en los criterios que bilateralmente se emiten, con el objetivo de llegar a una enseñanza comprensiva y potenciar la reflexión de las problemáticas que el maestro pone a disposición de alumnos de la carrera de Historia.

Se percibe que no todo está dicho en el salón de clases y para ello el docente potencia las diferentes miradas desde las que puede ser atendido un contenido. En su concepción se visualiza que posiciona al alumno como protagonista y para ello toma en cuenta lo que los une y los diferencia como estudiantes y sujetos sociales. Se evidencia que aunque planifica una clase donde se sugiere la interdependencia, no se observan actividades para desarrollar el trabajo colegiado entre los alumnos

En el caso de la intervención en la clase se enmarca en cómo desarrolla cada estrategia, donde retoma el acercamiento multidimensional de los elementos que presenta en el desarrollo de la misma, invitaba a emitir criterios, cuestionamientos y reflexiones muy particulares, en función de guiar el debate de manera flexible como se propone cuando se planea la clase. Se visualiza su compromiso para potenciar el rol como docente al rescatar información o conocimientos previos y el del alumno, al sugerir opiniones desde su experiencia y contextos de vida. 
Existe el respeto con los estudiantes al atender sus diferencias individuales, en función de que prime el compromiso social y ético, al buscarse un puente entre el sujeto y sus contextos. En diferentes momentos vincula los temas abordados en las clases con otras disciplinas que se trabajan en la carrera, tratando de no olvidar el equilibrio entre la reflexión y la rutina en el aula. A la vez se evidencian dificultades a la hora de concretar indicaciones de trabajo colegiado, a partir de la búsqueda de información y la solución de ejercicios en equipo. Es un asunto observado desde la planeación de la clase.

La evaluación de la clase se direcciona hacia valorar la participación en el debate y la reflexión sobre las problemáticas propuestas durante el desarrollo de la misma y para ello, el docente considera la generación de juicios y opiniones dadas, teniendo en cuenta las experiencias de cada uno de los alumnos. Considera como estrategia para evaluar, la realización de preguntas para reflexionar sobre el aprendizaje de contenidos. Asume que es pertinente tomar en cuenta el tipo de información y el juicio crítico que realizan de los contenidos expuestos. Sin embargo, como en anteriores momentos de la clase no se toma en cuenta el trabajo en equipo como criterio de evaluación.

\section{Conclusiones}

La práctica pedagógica reflexiva direcciona la mirada hacia una educación creativa, compleja que esté marcada por el pensamiento crítico, por el diálogo, la colegiabilidad, la interdisciplina y la transdisciplina. Se utiliza la teoría y el criterio de los maestros que participan con el objetivo de concretar un concepto marcado por estos elementos, para asociar creatividad y práctica reflexiva.
La construcción de las categorías creatividad del docente y práctica pedagógica reflexiva, supone el tránsito hacia una pauta que respete lo que los docentes creativos han defendido, donde no se critica un modelo determinado de hacer la docencia, sino que reclaman un acompañamiento en su búsqueda por adecuar su ejercicio profesional a la contemporaneidad y contextos; la intención es formar estudiantes reflexivos en el ámbito de la mutidimensionalidad del sistema social.

La investigación conlleva a identificar vacíos en la práctica pedagógica en su intención creativa e innovadora; se requiere la concepción de estrategias de mediación didáctica, donde el docente pueda evidenciar un perfil profesional centrado en la creatividad e innovación como cualidades intrínsecas, tanto a su componente personal como a su desempeño ocupacional y profesional.

Se concluye en la necesidad de consolidar una educación creativa sustentada en la resignificación de la práctica pedagógica, en función de los modos de actuación del docente y en el marco de las transformaciones sociocontextuales.

\section{REFERENCIAS}

Archila, G, J. (2013). Educación y pedagogía en el contexto del paradigma emergente: una nueva forma de pensar y percibir el mundo para la formación de ciudadanía. Revista Logos, Ciencia \& Tecnología, 5(1), 139-147. Disponible en https://www.redalyc.org/ pdf/5177/517751547011.pdf

Amabile, T. (1983). The social psychology of creativity: A componential conceptualization. Journal of personality and social psychology, 45(2), 357-376. https:// doi.org/10.1037/0022-3514.37.2.221 
Betancourt, M. (1997). Condiciones necesarias para propiciar atmósferas creativas. Revista Universal Universitaria, 10(12), 1-9. Recuperado de http://www. revista.unam.mx/vol.10/num12/art85/ art85.pdf

Cárdenas, N. y Angulo, F. (2016). Análisis de las dimensiones de adaptación, mejoramiento e innovación en los procesos de aprendizaje tecnológico. Cultura Educación y Sociedad, 7(2), 139-149. Recuperado a partir de https://revistascientificas.cuc.edu.co/culturaeducacionysociedad/article/view/1468

Caballero, E. (2012). La creatividad pedagógica en la formación del docente. Revista Didáctica y Educación, 3(4), 115-127. Disponible en https://dialnet.unirioja.es/servlet/ articulo?codigo $=4231952$

Castillejo, P., Pèrez-Alonso, M., Rodrìguez, T., Sarramon, J., Touriñan, J., Vazquez, G. (2012). Creatividad, educación e innovación: emprender la tarea de ser autor y no solo autor de sus propios proyectos . Revista de investigación en educación, 7-29.

Chibás-Ortiz, F. (2012). Creatividad + Dinámica de Grupo = Eureka. La Habana: Pueblo y Educación.

Cisterna, F. (2005). Categorización y Triangulación como Procesos de Validación del Conocimiento en Investigaciones Cualitativas. Theoria, 14(1) 6171. Disponible en https://www.redalyc. org/pdf/299/29900107.pdf

Csikszentmihalyi, M. (1998). Creatividad: el fluir y la psicología del descubrimiento y la invención. Barcelona: Paidós. Recuperado de https://campus.fundec.org.ar/admin/archivos/Mihaly_2.pdf

De Bono, E. (1986). El pensamiento lateral. Barcelona. Ediciones Paidós.
Dewey, J. (1933). How We Think: A Restatement of the Relation of Reflective Thinking to the Educative Process. Boston: D.C. Heath \& Co Publishers.

Guilford, J. y Strom, R. (1978). Creatividad y Educación. Buenos Aires. Paidós.

López, L. (2015). Conocimiento, pensamiento complejo y universidad. Revista Cubana de Educación Superior. 34(2). 28-33. Recuperado de http://scielo.sld. cu/scielo.php?script=sci_arttext\&pid $=$ S0257-43142015000200003

Marín, F., Cabas, J., Cabas, L. y Paredes, A. (2018). Formación Integral en Profesionales de la Ingeniería. Análisis en el Plano de la Calidad Educativa. Formación Universitaria, 11(1), 13-24. http://dx.doi.org/10.4067/S071850062018000100013.

Martínez, M. (2009). Maestro y creatividad ante el siglo XXI. En, M. Martínez Llantada y Guanche, A. (Compil.). En, El desarrollo de la creatividad. Teoría y práctica en la educación (pp. 109-116). La Habana: Pueblo y Educación.

Maldonado, C. (2014). ¿ Qué es eso de pedagogía y educación en complejidad. Intersticios sociales, 7(1), 1-23. Disponible en https:/www.redalyc.org/ pdf/4217/421739500002.pdf

Monsalve, M. (2015). Paradigma de la complejidad en educación. Atlante: Cuadernos de Educación y Desarrollo. Disponible en: http://www.eumed.net/ rev/atlante/2015/12/escuela.html

Morin, E. (2003). Introducción al pensamiento complejo. Barcelona: Gedisa

Mujica, M., Marín, F. Smith, H. y Lovera, M. (2008). Municipio innovador, un modelo para el desarrollo local sostenible. Multiciencias, 8(Extraordinario), 130-136. Disponible en https://www. redalyc.org/pdf/904/90411691019.pdf 
Remedios, J. M. (2016). Pedagogía para el desarrollo de la creatividad en educación y para la educación. Lima: Magisterial.

Schön, D. (2010). La formación de profesionales reflexivos: hacia un nuevo diseño de la enseñanza y el aprendizaje en las profesiones. Madrid: Paidós.

Sternberg, R. y Lubart, T. (1997). La creatividad en una cultura conformista. Un desafio las masas. Madrid: Paidós.

Torrance, E. (1965). Opiniones científicas de la creatividad y factores que afectan su crecimiento. Dédalo, 94(3), 663681. 